http://jmscr.igmpublication.org/home/ ISSN (e)-2347-176x ISSN (p) 2455-0450 crossref DOI: https://dx.doi.org/10.18535/jmscr/v9i8.12

\title{
Susceptibility of Gram negative isolates to Nitrofurantoin in Urinary Tract Infection at a tertiary health care center in Himachal Pradesh
}

\author{
Authors \\ Dr Aditya Rana', Dr Subhash Chand Jaryal'², Dr Anuradha Sood ${ }^{3}$, \\ Dr Meenakshi Tamrakar ${ }^{4}$, Dr Anjali Sharma ${ }^{5}$ \\ ${ }^{1} \mathrm{JR}$, Department of Microbiology, DRPGMC Kangra at Tanda \\ ${ }^{2}$ Professor and Head of the department, Department of Microbiology, DRPGMC Kangra at Tanda \\ ${ }^{3}$ Associate Professor, Department of Microbiology, DRPGMC Kangra at Tanda \\ ${ }^{4} \mathrm{JR}$, Department of Microbiology, DRPGMC Kangra at Tanda \\ ${ }^{5} \mathrm{JR}$, Department of Microbiology, DRPGMC Kangra at Tanda
}

\begin{abstract}
Introduction: The most common bacterial infection affecting human beings in life time is Urinary tract infections (UTI). Escherichia coli (E.coli) is the most common pathogen in UTIs. Nitrofurantoin has been used for a long time. In era of increasing resistance and the decline in newly developed antibiotics has escalated engrossment in treatment of bacterial UTI.

Materials \& Methods: A descriptive analysis of culture results of urine samples was performed at the Department of Microbiology at DRPGMC Kangra at Tanda from May 2020 to April 2021. Samples were sent in a sterile wide mouth container. The specimens were inoculated on MacConkey agar plates and incubated aerobically at $37^{\circ} \mathrm{C}$ for 24 hours. Culture plates with colony counts of $\geq 10^{5} \mathrm{cfu} / \mathrm{ml}$ were considered positive for UTI. Antimicrobial susceptibility was performed according to CLSI guidelines.

Results: A total of 4286 samples were received, out of which 1244(29.1\%) were males and 3042 (70.9\%) females. Male to female ratio was 1:2.4. Majority of the cases (71.2\%) belonged to the age group 25-45 years. Escherichia coli (E.coli) (50.7\%) was the most common pathogen among Gram negative isolates. Maximum sensitivity was seen in E.coli (92\%) and maximum resistance was seen in Acinetobacter (90\%)

Conclusion: E.coli remained the predominant isolate among gram negative organisms, more commonly in females. The in vitro activity of nitrofurantoin found in the present study suggests that this drug is susceptible to most uropathogens.Regular antimicrobial susceptibility is required to be carried out to see the current pattern of susceptibility.

Keywords: Urinary tract infection, Nitrofurantoin, Sensitivity, Susceptibility, Resistance.
\end{abstract}

\section{Introduction}

The most common bacterial infection affecting human beings in life time is Urinary tract infections (UTI). They are the frequent cause of morbidity in outpatients as well as most frequently involved in the cause of Healthcare associated infection $^{[1]}$. Due to marginal discovery of newer antibacterial agents entering clinical practice, resistance is widely recognized as a major threat to public health sectors. ${ }^{[2]}$

Nitrofurantoin has been used for decades as an alternative treatment of uncomplicated UTIs. 
Nitrofurantoin has $80 \%$ oral bioavailability, and approximately $25 \%$ is excreted unchanged in the urine, with only a small portion reaching the colon. ${ }^{[3]}$ Additionally, nitrofurantoin has retained a high prevalence of sensitivity to most uropathogens and has a favorable side-effect profile $^{[4]}$. Nitrofurantoin is a broad spectrum bactericidal antibiotic that, through a complex mode of action which is not completely understood, affects both gram negative and positive bacteria. ${ }^{[5]}$

Nitrofurantoin has been used for a long time, but the emergence of antimicrobial resistance and the decline in newly developed antibiotics has escalated engrossment in treatment of bacterial UTI with this antibiotic. Resistance to nitrofurantoin remained virtually unchanged since its discovery. ${ }^{[6]}$

Decisive role of nitrofurantoin is crucial in the era of increasing resistance in uropathogens. Antimicrobial resistance pattern is required to trace any change that might have occurred in time so that updated recommendations for optimal empirical therapy of UTI can be made.

\section{Materials and Methods}

Study design, a descriptive retrospective analysis of culture results of urine samples was performed at Microbiology department of Microbiology, DRPGMC Kangra at Tanda, Himachal Pradesh from May 2020 to April 2021. The sex and age of patients, the organism isolated and the antimicrobial susceptibility profiles were collected from the registration records using a standard data collection form.

The samples were received in the Department of Microbiology from both inpatient and outpatient departments and included male and female patients.

\section{Culture and Identification}

Specimens were received in sterile wide mouth universal containers. Samples were processed on the same day. A semi quantitative method was adopted for primary isolation of organisms using a calibrated loop of $4 \mathrm{~mm}$ diameter which delivers $10 \mu 1$ of urine ${ }^{[7]}$. The specimens were inoculated on Mac Conkey agar plates and incubated aerobically at $37^{\circ} \mathrm{C}$ for $24-48$ hours. Culture plates with colony counts of $\geq 10^{5} \mathrm{cfu} / \mathrm{ml}$ were considered positive for UTI. Cultures that showed no growth in 24 to 48 hours indicated absence of infection as sterile. From positive cultures, uropathogens were identified according to the standard biochemical reactions ${ }^{[8]}$. A significant bacterium was considered if urine culture yielded $\geq 10^{5} \mathrm{CFU} / \mathrm{ml}$ and $<10^{5} \mathrm{CFU} / \mathrm{ml}$ is taken as insignificant. ${ }^{[9]}$

\section{Antimicrobial susceptibility testing}

According to the standard operational procedures, in vitro antimicrobial susceptibility testing was done on Mueller-Hinton agar (Hi-Media Lab Ltd, India) using Kirby-Bauer disc diffusion method. A suspension of the test organism was made in sterile normal saline and turbidity adjusted to 0.5 McFarland standards. The test organism was uniformly seeded over the surface of Mueller Hinton agar plates. The plates were allowed to dry for 10 minutes before application of antibiotic discs. The plates were incubated at $37^{\circ} \mathrm{C}$ for $16-18$ hours. After incubation clear zones around the antibiotic discs were measured with a ruler and recorded in millimeters. Nitrofurantoin $(300 \mu \mathrm{g})$ antibiotic disc was used. Susceptibility and resistance data was interpreted according to Clinical laboratory Standards Institute guidelines. ${ }^{[10]}$

\section{Results}

A total of 4286 urine specimens were received in the Department of Microbiology for the duration of 1 years from May 2020 to April 2021. Out of which $1320(30.8 \%)$ were from OPD and 2966 $(69.2 \%)$ from IPD. 1244(29.1\%) were males and $3042(70.9 \%)$ females. Male to female ratio 1:2.4. Majority of the cases $(71.2 \%)$ belonged to the age group 25-45 years. (Table 1)

Table 1: Gender distribution

\begin{tabular}{|l|c|c|}
\hline Gender & Number & Percentage(\%) \\
\hline Male & 1244 & $29.1 \%$ \\
\hline Female & 3042 & $70.9 \%$ \\
\hline
\end{tabular}


The culture positivity rate observed in this study was $15.6 \%$ (672 out of 4286 samples). Out of 672, gram negative isolates were 627 (93.3\%), 33 (4.9\%) were Gram positive isolates and 12 $(1.78 \%)$ candida species. 239(5.6\%) samples had insignificant growth i.e $<10^{5} \mathrm{cfu} / \mathrm{ml} .3615(84.3 \%)$ out of 4286 samples were sterile.
Escherichia coli (E.coli) (50.7\%) were the most common pathogen among Gram negative isolates followed by Klebsiella sps. (14.1\%) (Table 2). S.aureus $(4.9 \%)$ was the most common pathogen among Gram positive isolates.

Table 2: Gram negative isolates from urine sample

\begin{tabular}{|l|c|c|c|}
\hline S.r No. & Gram negative isolates & Number & Percentage (\%) \\
\hline 1 & Escherichia coli & 318 & 50.7 \\
\hline 2 & Klebsiella sps & 89 & 14.1 \\
\hline 3 & Acinetobacter sps & 58 & 9.2 \\
\hline 4 & Pseudomonas sps & 54 & 8.6 \\
\hline 5 & Non fermenter group & 45 & 7.1 \\
\hline 6 & Citrobacter & 38 & 6 \\
\hline 7 & Proteus sps & 21 & 3.3 \\
\hline 8 & Morganella sps & 4 & 0.6 \\
\hline
\end{tabular}

Susceptibility to Nitrofurantoin was least in Acinetobacter $90 \%$, followed by Pseudomonas $88.4 \%$ and Proteus (85.7.\%) (Table 3)

Table 3: Antimicrobial resistant pattern of Nitrofurantoin to various isolates

\begin{tabular}{|l|c|c|c|c|}
\hline Sr.No. & Gram negative isolates & Susceptible to Nitrofurantoin & Resistant to Nitrofurantoin & Total \\
\hline 1 & Escherichia coli & $293(92 \%)$ & $25(8 \%)$ & 318 \\
\hline 2 & Klebsiella sps & $43(48.3 \%)$ & $46(51.6 \%)$ & 89 \\
\hline 3 & Acinetobacter sps & $6(10 \%)$ & $52(90 \%)$ & 58 \\
\hline 4 & Pseudomonas sps & $6(11.1 \%)$ & $48(88.8 \%)$ & 54 \\
\hline 5 & Non fermenter group & $14(31.1 \%)$ & $31(68.8 \%)$ & 45 \\
\hline 6 & Citrobacter sps & $20(52.6 \%)$ & $18(47.3 \%)$ & 38 \\
\hline 7 & Proteus sps & $3(14.2 \%)$ & $18(85.7 \%)$ & 21 \\
\hline 8 & Morganella & $4(100 \%)$ & $0(0 \%)$ & 4 \\
\hline 9 & Total & $389(62 \%)$ & $238(38 \%)$ & 627 \\
\hline
\end{tabular}

\section{Discussion}

With the increases in antibiotic resistance among Enterobacterales and other gram negative isolates over the past several decades, surveillance data have become critical for appropriate empiric selection of antibiotic therapy. Antibiotic treatment is typically selected empirically, based on the patient clinical presentation, medical history and local patterns of antibiotic susceptibility ${ }^{[11]}$.

In the present study gram negative pathogens $627(93.3 \%)$ outnumbered gram positive organisms $33(4.9 \%)$ similar results were seen in study by Khoshbakht $\mathrm{R}$ et al who reported predominance of gram negative bacilli $(83.17 \%)$ while gram positive organisms as $21.73 \%^{[12]}$. Among gram negative isolates and
Enterobacterales Escherichia coli remained predominant isolate $(50.7 \%)$ which is comparable to findings of Khoshbakht $\mathrm{R}$ et al , Shalini et al 2011 and Kibret et al who also reported Escherichia coli as most predominant pathogen isolated from urine samples with prevalence of $66.08 \%, 64.33 \%$ and $63.6 \%$ respectively ${ }^{[12,13,14]}$.

Majority of Escherichia coli isolates were susceptible to Nitrofurantoin $92 \%$, with resistant isolates only $8 \%$, which is similar to results documented by Shalini et al ,Kibret et al and Rijal A et al 2012, in which sensitive isolates were $93.48 \%, 96.2 \%$ and $96.5 \%$ with only $6.52 \%, 3.8 \%$ and $3.5 \%$ resistant isolates ${ }^{[13,14,15]}$.

The drug of choice for E.coli as depicted by the findings of present study remains Nitrofurantoin as $92 \%$ isolates were sensitive, with only $8 \%$ 
isolates resistant. Resistance was mainly seen with Acinatobacter (90\%), Pseudomonas (88.8\%) and Proteus $(85.7 \%)$. Similar in a study by Rachna et al showed only $6.6 \%$ susceptibility of Acinetobacter to nitrofurantoin. ${ }^{[16]}$ In a study by Grayson et al showed resistance of nitrofurantoin to Pseudomonas and Acinetobacter. ${ }^{[17]}$ Reason for resistance in Proteus species, and Pseudomonas aeruginosa is that it is naturally resistant to nitrofurantoin $^{[18,19]}$ In our study overall susceptibility of nitrofurantoin in enterobacterale was high. This may be due to Nitrofurantoin's narrow spectrum of activity, limited indication, narrow tissue distribution, and limited contact with bacteria outside the urinary tract ${ }^{[20]}$.

\section{Conclusion}

Escherichia coli remained the predominant isolate among enterobacterales and gram negative organisms, more commonly in females. This study shows that nitrofurantoin was found to be susceptible in most of the uropathogens. As it is a sparing drug it should be used wisely. Nitrofurantoin was found to be resistant in Acinetobacter, Proteus, Pseudomonas and non fermenter isolates. A review of antibiotic policy pertaining to treatment of urinary tract infections is necessary. Regular antimicrobial susceptibility is required to be carried out to see the current pattern of susceptibility. Empirical treatment policies should be prepared and common working policy has to be formulated using local surveillance data to guide the empiric selection of antibiotic therapy as well as prevention of development of resistance.

\section{Bibliography}

1. HauslerJr, Sussman M. Urinary Tract Infections. Topley and Wilson's Microbiology and Microbial Infections. 9th ed., Arnold; 1998: 601-21.

2. Coskun O, Erdem H, Avcı A. Management of community-acquired acute bacterial cystitis in Turkey. Turk J Med Sci 2011; 41(1):149-57.
3. Gardiner BJ, Stewardson AJ, Abbott IJ, Peleg AY. Nitrofurantoin and fosfomycin for resistant urinary tract infections: old drugs for emerging problems. Aust Prescr. 2019;42(1):14-9.

4. Spencer RC, Moseley DJ, Greensmith MJ. Nitrofurantoin modified release versus trimethoprim or co-trimoxazole in the treatment of uncomplicated urinary tract infection in general practice. J Antimicrob Chemother. 1994 May;33 Suppl A:121-9.

5. Munoz-Davila MJ. Role of Old Antibiotics in the Era of Antibiotic Resistance. Highlighted Nitrofurantoin for the Treatment of Lower Urinary Tract Infections. Antibiotics (Basel). $2014 \mathrm{Feb}$ 10;3(1):39-48.

6. Neelima A, Kiranmai. Nitrofurantoin susceptibility of ESBL gram negative isolates from patients with urinary tract infection (UTI) in a rural teaching hospital of Telangana.Trop $\mathrm{J}$ Path Micro 2016;2(3):159-163.

7. Ozlem KA Hande A. Risk factors for ciprofloxacin resistance among Escherichia coli strains isolated from community acquired urinary tract infections in turkey. $\mathrm{J}$ Antimicrobial Chemother. 2005; 56(5):914-918.

8. J.G. Colle, R.S. Miles, B.Watt. Tests for Identification of bacteria. Mackie and Mc Cartney Practical Medical Microbiology 14th edition, Churchill Livingstone 2008: P131-149.

9. KASS EH. Bacteriuria and the diagnosis of infections of the urinary tract; with observations on the use of methionine as a urinary antiseptic. AMA Arch Intern Med. 1957 Nov;100(5):709-14.

10. Clinical Laboratory Standard Institute. Performance Standards for Antimicrobial Susceptibility Testing; Twenty-Second Informational Supplement. Vol. 32. Clinical Laboratory Standard Institute; 
Wayne, Pennsylvania, USA: 2012. pp. 7071.

11. Gupta K, Hooton TM, Naber KG, Wullt B, Colgan R, Miller LG et al. International clinical practice guidelines for the treatment of acute uncomplicated cystitis and pyelonephritis in women: A 2010 update by the Infectious Diseases Society of America and the European Society for Microbiology and Infectious Diseases. Clin Infect Dis 2011; 52(5): 103-20.

12. Khoshbakht R, Salimi A, Aski HS, Keshavarzi H. Antibiotic susceptibility of bacterial strains isolated from urinary tract infections in Karaj,Iran. Jundishapur $\mathbf{J}$ Microbiol 2013; 6(1):86-90.

13. Shalini, Joshi MC, Rashid MK, Joshi HS. Study of Antibiotic Sensitivity Pattern in Urinary Tract Infection at a Tertiary Hospital. Nat J Integr Res Med 2011; 2(3):43-6.

14. Kibret M, Abera B. Prevalence and antibiogram of bacterial isolates from urinary tract infections at Dessie Health Research Laboratory, Ethiopia.Asian Pac J Trop Biomed 2014; 4(2): 164-68.

15. Rijal A, Ghimire G, Gautam K, Barakoti A. Antibiotic Susceptibility of Organisms Causing Urinary Tract Infection in Patients Presenting to a Teaching Hospital. J Nepal Health Res Counc 2012; 10(20):24-27.

16. Tewari R, Chopra D, Wazahat R, Dhingra S, Dudeja M. Antimicrobial susceptibility patterns of an emerging multidrug resistant nosocomial pathogen: Acinetobacter baumannii. Malays J Med Sci. 2018;25(3):129-134.

17. Grayson ML, Cosgrove SE, Crowe SM, Hope W, Mccarthy JS, Mills J, et al., editors. Kucers' the use of antibiotics: a clinical review of antibacterial, antifungal, antiparasitic and antiviral drugs. 7th ed. Boca Raton (FL): CRC Press; 2017.

18. Brumfitt W, Hamilton-Miller MT. Efficacy and safety profile of long-term nitrofurantoin in urinary infections: 18 years' experience. $J$ Antimicrob Chemother. 1998;42:363-371.

19. Grayson ML, Whitby M. Nitrofurans: nitrofurazone, furazolidone, and nitrofurantoin. In: Grayson ML, Crowe SM, McCarthy JS, et al., eds. Kucers' The Use of Antibiotics. 6th ed. London, England: Hodder Arnold; 2010:1195-1204 20. James AK, Laurie J, Clyde T, Mark EJ, Daniel FS. Trends in Antimicrobial Resistance among Urinary Tract Infection Isolates from Female Outpatients in the United States. Antimicrob Agents Chemother 2002; 46(8): 2540-45. 